\title{
Article \\ Postoperative Stability of Patients Undergoing Orthognathic Surgery with Orthodontic Treatment Using Clear Aligners: A Preliminary Study
}

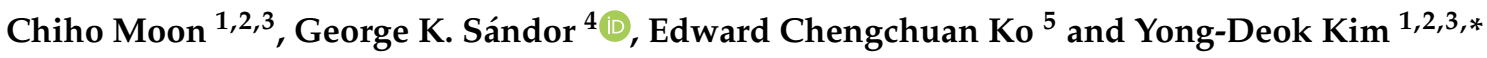 \\ 1 Department of Oral and Maxillofacial Surgery, School of Dentistry, Pusan National University, Geumo-ro 20, \\ Mulgeum, Yangsan 50612, Korea; mch2875@naver.com \\ 2 Dental and Life Science Institute, School of Dentistry, Pusan National University, Yangsan 50612, Korea \\ 3 Dental Research Instutite, Pusan National University Dental Hospital, Yangsan 50612, Korea \\ 4 Department of Oral and Maxillofacial Surgery, Oulu University Hospital, University of Oulu, \\ 90220 Oulu, Finland; sandor_george@hotmail.com \\ 5 Department of Oral and Maxillofacial Surgery, Kaohsiung Medical University, Kaohsiung 80708, Taiwan; \\ ko.edward.kaseizen@gmail.com \\ * Correspondence: ydkimdds@pusan.ac.kr; Tel.: +82-55-360-5116; Fax: +82-55-360-5104
}

check for

updates

Citation: Moon, C.; Sándor, G.K.; Ko, E.C.; Kim, Y.-D. Postoperative Stability of Patients Undergoing Orthognathic Surgery with Orthodontic Treatment Using Clear Aligners: A Preliminary Study. Appl. Sci. 2021, 11, 11216. https://doi.org/ 10.3390/app112311216

Academic Editors: Gabriele Cervino and Dorina Lauritano

Received: 2 November 2021

Accepted: 24 November 2021

Published: 25 November 2021

Publisher's Note: MDPI stays neutral with regard to jurisdictional claims in published maps and institutional affiliations.

Copyright: (c) 2021 by the authors. Licensee MDPI, Basel, Switzerland. This article is an open access article distributed under the terms and conditions of the Creative Commons Attribution (CC BY) license (https:/ / creativecommons.org/licenses/by/ $4.0 /)$.

\begin{abstract}
Backgrond: Until now, there have been many studies on the postoperative stability of orthognathic patients treated with traditional fixed orthodontic appliances. Recently, the use of clear aligners as orthodontic appliances has increased in orthodontic treatment for aesthetic and patient convenience. The aim of this preliminary study was to investigate the stability and characteristics of patients undergoing orthognathic surgery using clear aligners. Patients and Methods: This study was performed on patients who underwent orthognathic surgery by one surgeon at Pusan National University Dental Hospital from April 2017 to August 2021. A comparative study was conducted on five patients treated with clear aligners during orthodontic treatment and ten patients treated with traditional fixed appliances as a control group. Postoperative skeletal changes and recurrence were evaluated by cone beam computed tomography and lateral cephalometric radiographs taken two days postoperatively and six months postoperatively. Several measurement variables were used to confirm the presence or absence of recurrence, preoperative and postoperative orthodontic treatment period, and the number of extracted teeth. Results: Postoperative stability for six months after surgery was not significantly different between the clear aligner group and the traditional fixed appliance group. The preoperative orthodontic treatment period was also shorter in the clear aligner group, and the number of extracted premolar teeth and impacted teeth were also fewer in the clear aligner group, but there was no significant difference. Conclusions: Orthodontic treatment using clear aligners continues to develop, and it is believed that there is no limit to what can be accomplished during orthodontic surgery accompanied by clear aligners.
\end{abstract}

Keywords: orthognathic surgery; clear aligner; Invisalign; stability; preoperative orthodontic treatment; CAD-CAM; 3D printer

\section{Introduction}

Since Kesling first invented the aligner in 1946, an aligner for tooth alignment has been developed [1]. Recently, patient demand has increased for more orthodontic treatments with esthetic appliances [2]. In line with this, Align Technology Inc. (San Jose, CA, USA) launched the Invisalign system in 1999, which enables esthetic orthodontic treatment because traditional braces are not invisible. This was the first CAD (computer-aided design) and CAM (computer-aided manufacturing)-based orthodontic appliance, which can be digitally scanned, and made as a series of aligners using a single impression [3,4].

The approximate digital manufacturing process of a clear aligner is as follows. First, a digital image is obtained by scanning a plaster model taken either by direct impression 
of the patient's dentition with a three-dimensional (3D) scanner or by directly scanning the patient's dentition with an intraoral scanner. When the digital model is created in this way, the file can be loaded in the setup program to start making the clear aligner $[4,5]$. Following the setup program, the crown, root, and long axis are set, and the teeth are moved to the desired position, which is the final arrangement. After that, a series of clear aligners suitable for the treatment period is designed. At this stage, it is recommended that the tooth rotation is within 2.5 degrees and the movement distance confined to $0.3 \mathrm{~mm}$ at each stage [6]. After that, a 3D printer is used to make a model for step-by-step changes and then make a series of progressive clear aligners to move the misaligned teeth.

Recently, increasing numbers of patients are receiving orthodontic treatment with clear aligners, including those patients undergoing orthognathic surgery. Although there have been many studies showing that the postoperative stability of orthodontic patients treated with conventional fixed orthodontic devices is good $[7,8]$, there have been no studies on postoperative stability of orthodontic patients treated with clear aligners. In this preliminary study, the stability and characteristics of patients using clear aligners after orthognathic surgery were investigated.

\section{Materials and Methods}

\subsection{Patients}

This retrospective study was exempt from review by the Institutional Review Board at Pusan National University Dental Hospital (Approval No.: PNUDH-2021-038). A total of 15 patients who underwent orthognathic surgery by one surgeon at PNUDH between April 2017 and August 2021 were included in this study. Of the 15 patients, 5 patients received preoperative orthodontic treatment with clear aligners, and the remaining 10 patients received orthodontic treatment with traditional fixed appliances.

\subsection{Study Methods}

For all patients, cone beam computed tomography (CBCT), and lateral cephalometric radiographs were taken postoperatively on day 2 and month 6 to confirm skeletal changes (Figures 1 and 2). Postoperative stability was evaluated and analyzed by measuring several measurement variables (L1 to NB, Pog. to NB, A-Pog., angle of facial convexity (AFC), SN vertical, and 0-merdian) (Table 1). The duration of each patient's preoperative orthodontic period, the number of premolar extractions, and the number of impacted teeth were also compared and analyzed.

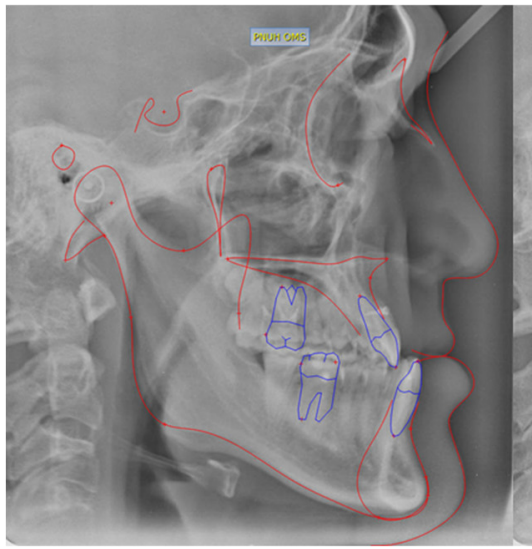

(a)

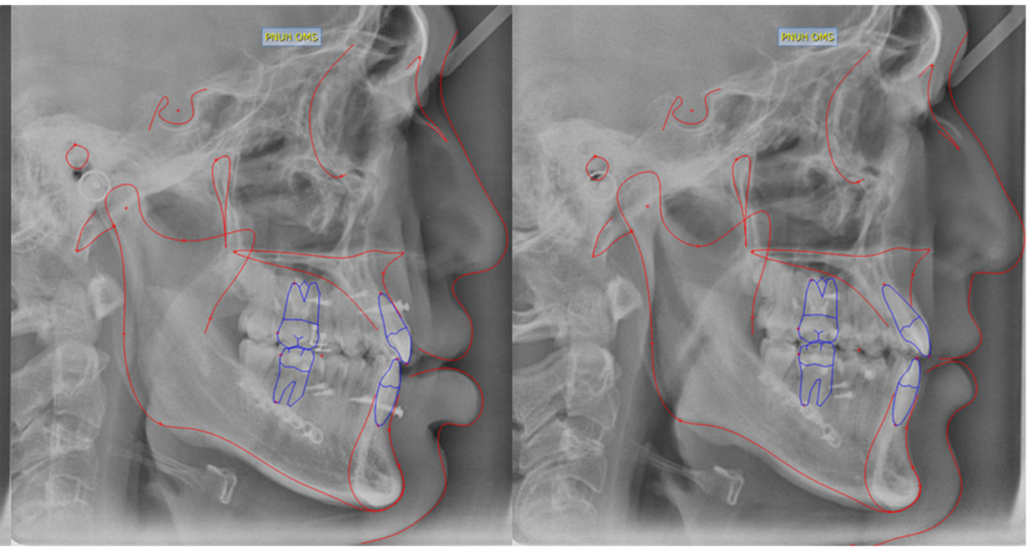

(b) (c)

Figure 1. Orthognathic surgery patient with clear aligners. (a) Before surgery; (b) 2 days after surgery; (c) 6 months postoperative radiograph image. Braces attached to teeth were not observed on radiographs. 


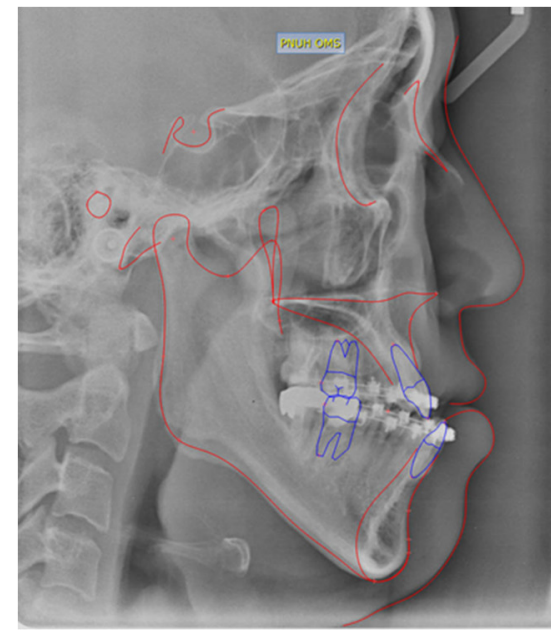

(a)

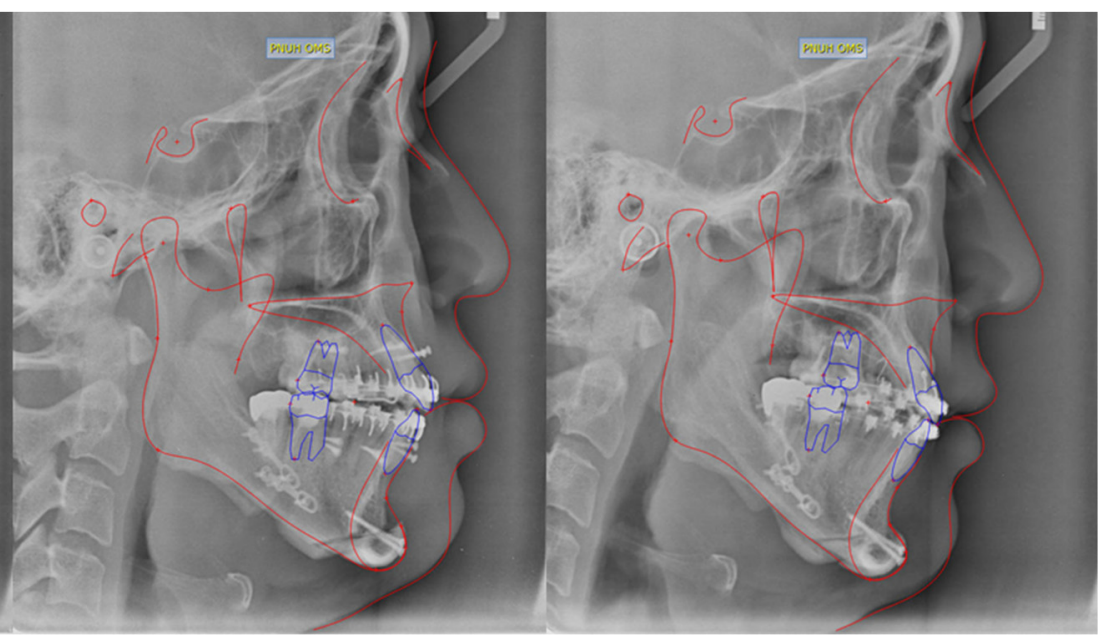

(b) (c)

Figure 2. Orthognathic surgery patient with conventional fixed appliance. (a) Before surgery; (b) 2 days after surgery; (c) 6 months postoperative radiograph image. The bracket attached to teeth was not observed on radiographs.

Table 1. Measurement variables.

L1 to NB (linear) (mm): Distance from [Mandible 1 crown] to Line [B-point]-[Nasion]

Pog. to NB (mm): Distance from [Pogonion] to Line [B-point]-[Nasion] Positive for right side of Line

A-Pog. (mm): Distance from [Mandible 1 crown] to Line [A-Point]-[Pogonion] Positive for right side of Line

Angle of facial convexity (AFC) (deg): Angle of line [Subnasale]-[Glabella] and [soft tissue Pogonion]-[Subnasale]

SN Vr. (mm): Subtract distance of [Subnasale] from distance of [soft tissue Pogonion], parallel to line [Porion]-[Orbitale]

0-Med. (mm): Subtract distance of [soft tissue Nasion] from distance of [soft tissue Pogonion], parallel to line [Porion]-[Orbitale]

\subsection{Statistical Analysis}

All measurements were statistically analyzed using a statistical program (SPSS 14.0; IBM SPSS Statistics, Chicago, IL, USA). All landmarks were analyzed using a cephalometric analysis program (Vceph 6.0; Osstem implant Co., Seoul, Korea)

\subsection{The Manufacturing Process of Clear Aligners by CAD/CAM}

In our hospital, the patient's dentition is obtained directly from the oral cavity using an intraoral scanner (Figure 3). Following this, the digital model is called from the setup program. Common programs include Maestro 3D Ortho Studio (Age solutions, Pontedera, Italy), Ortho Analyzer (3Shape, Copenhagen K, Denmark), Orchestrate 3D (Orchestrate 3D, Rialto, CA, USA) [5], and Autolign (Dorico, Yongin, Korea). In this study, the Autolign (Dorico, Yongin, Korea) program was used. Each tooth number is marked on the imported digital model, and the most mesial and distal points of each tooth are marked (Figure 4). After that, the cutting line of the crown is corrected to accurately mark the clinical crown (Figure 5). Next, when the tooth axis and root are formed, the model setup is completed (Figure 6). After that, the teeth are moved to the desired final occlusion (Figure 7). At this time, each tooth can move not only in parallel but also by rotation (Figure 8). When the final occlusion is set, the movement amount (within $0.3 \mathrm{~mm}$ ) [5] and rotation of each step is set within 2.5 degrees [6], and after deciding how many steps to make for the clear aligners (Figure 9), the series of models are printed using a 3D printer. After that, clear aligners are manufactured using a pressure molding machine for each model in the series. 

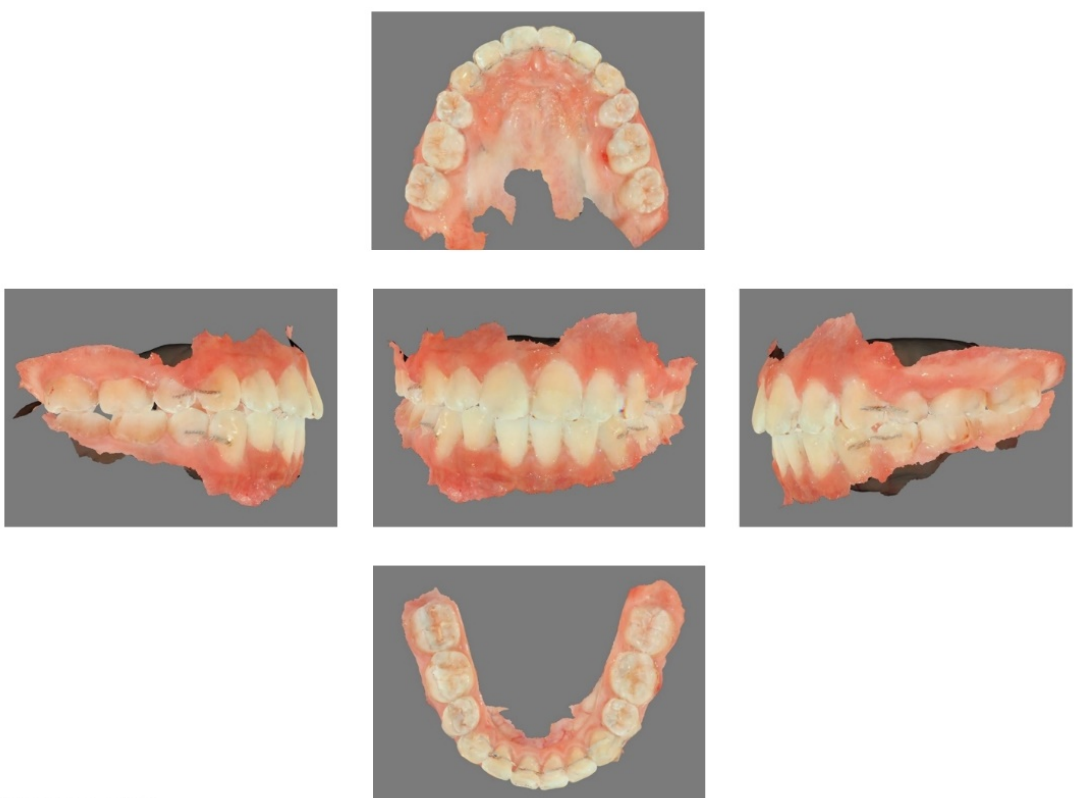

Figure 3. Photo taken directly from intraoral dentition using an intraoral scanner. First, each member of the dentition of the maxilla and mandible is scanned, then the patient is occluded, and then the current occlusion is also scanned.

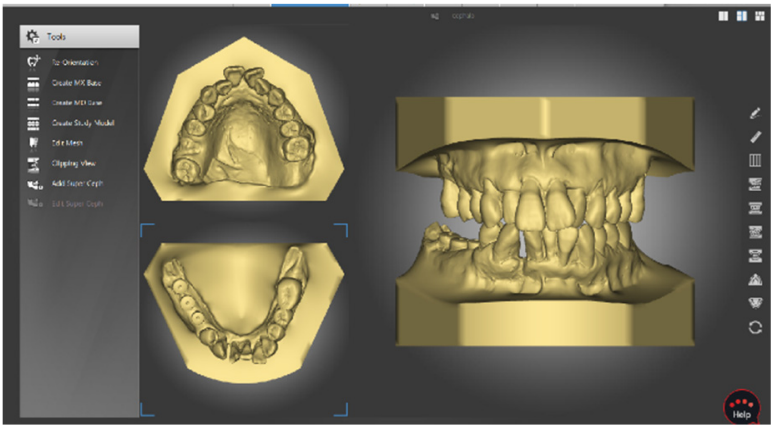

(a)

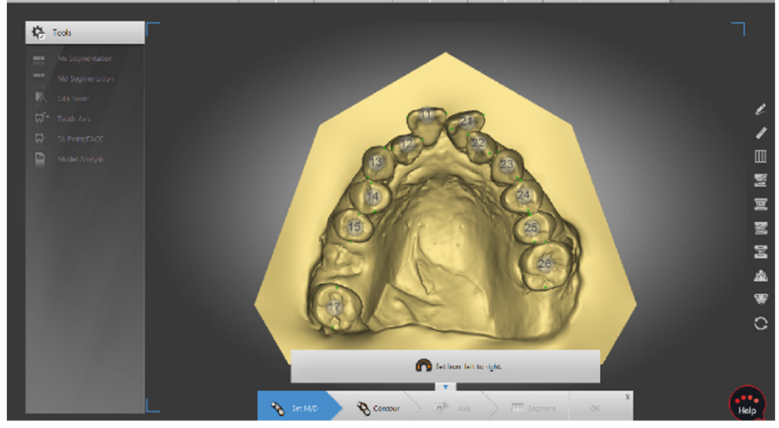

(b)

Figure 4. (a) The screen where the digital model is loaded into the setup program. One can see the dentition of the upper and lower jaws and the patient's current occlusion status. (b) A photograph showing the most mesial and distal parts of each tooth with green dots after each tooth marked to distinguish and display the teeth in the digital model.

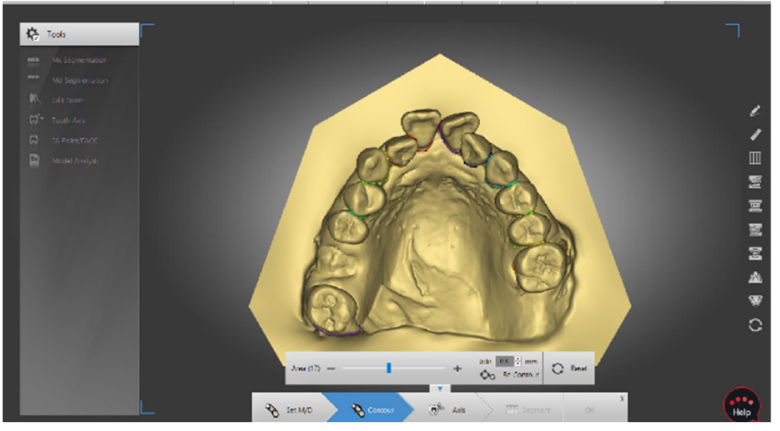

(a)

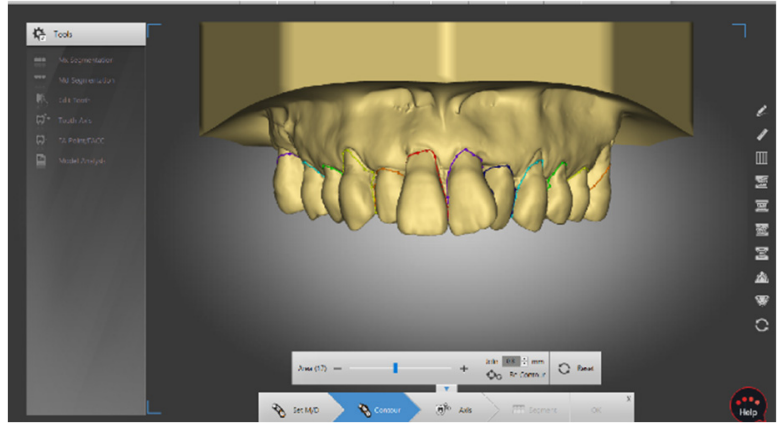

(b)

Figure 5. (a) In order to more accurately realize the crown of each tooth, the screen is curved by placing additional dots between the markings of each tooth performed in Figure $4 \mathrm{~b}$. The degree of curvature is also freely adjustable. The gingival margin and the boundaries between the teeth are precisely marked. (b) The screen of (a) viewed from the front. 


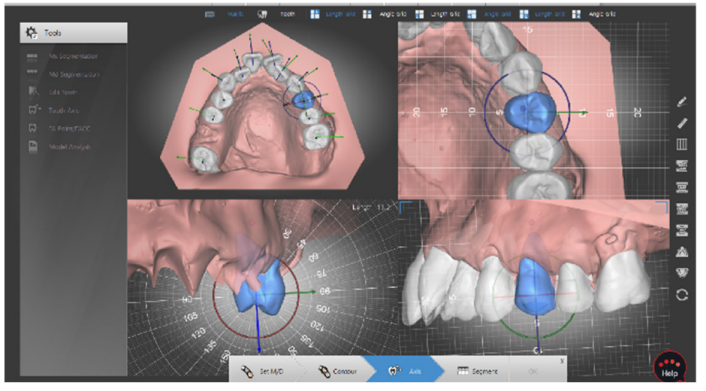

(a)

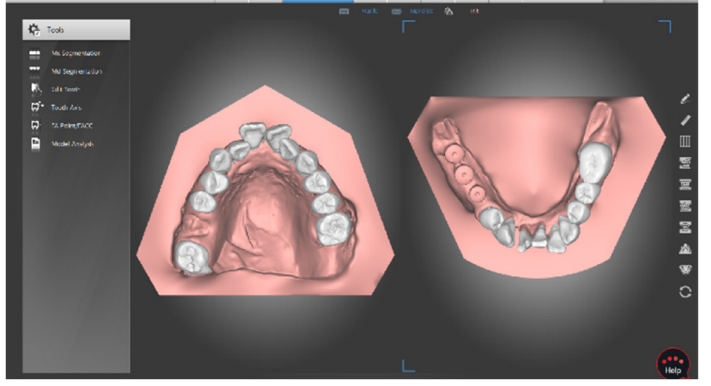

(b)

Figure 6. (a) A step in which a virtual root is formed by marking the major axis of each tooth. At this time, it is possible to rotate in mesio-distal, bucco-palatal (bucco-lingual), and clockwise/counterclockwise rotation of the teeth when viewed from the occlusal plane. (b) A step in which both the crown and root are set up. The patient's existing dentition has been accurately implemented.

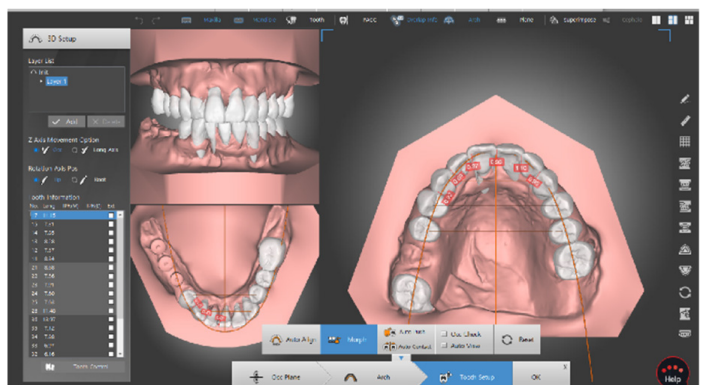

(a)

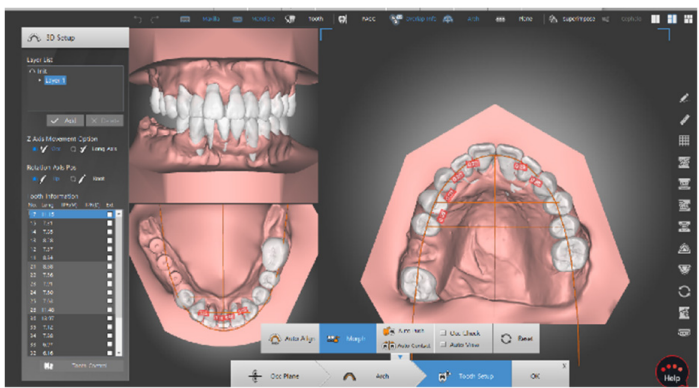

(b)

Figure 7. $(\mathbf{a}, \mathbf{b})$ Screen for moving each tooth to the final occlusion. It is possible to check the amount of movement of each tooth when the teeth are moved from the existing dental state to the final occlusion.

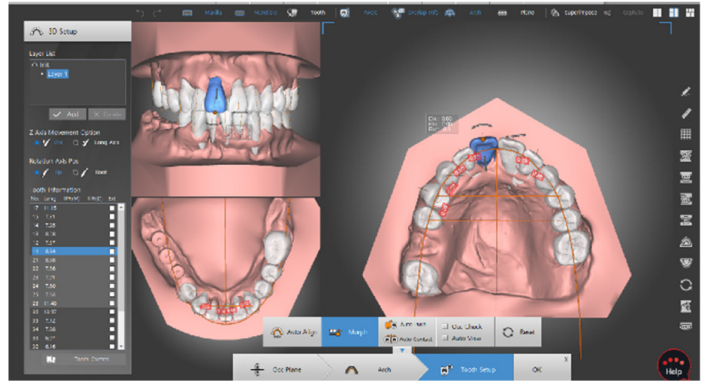

(a)

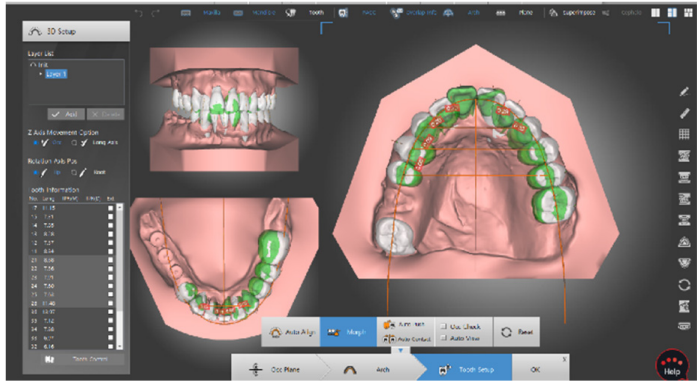

(b)

Figure 8. (a) Photograph showing that each tooth can rotate and move parallel to the bucco-palatal (bucco-lingual) and mesio-distal. (b) A photograph of overlapping existing occlusion (green) and final occlusion (white).

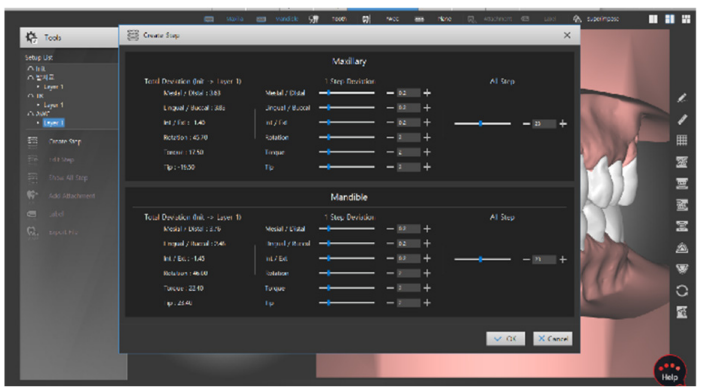

(a)

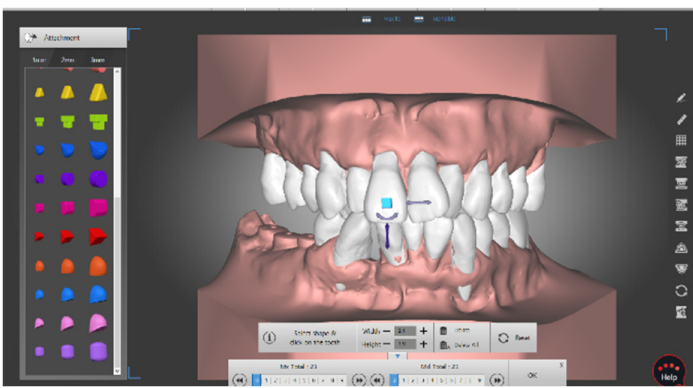

(b)

Figure 9. (a) A screen with a rotation of 2 degrees within one step, a movement distance of $0.2 \mathrm{~mm}$, and tooth movement planned in 23 steps. (b) If necessary, an attachment can be added to the tooth. 


\section{Results}

Of the 15 patients ( 11 males, 4 females, mean age: $22.6 \pm 4.36), 5$ patients ( 5 males, 0 females, mean age: $22.2 \pm 1.33$ ) received preoperative orthodontic treatment with clear aligners, and the remaining 10 patients ( 6 males, 4 females, mean age: $24.0 \pm 7.86$ ) received orthodontic treatment with traditional fixed appliances as a control group. Only one patient in the clear aligner group underwent Le Fort I osteotomy and mandibular bilateral sagittal split osteotomy surgery, and the remaining 14 patients had only mandibular bilateral sagittal split osteotomy surgery.

There was no significant difference between the two groups, although the preoperative orthodontic period was longer in those who received orthodontic treatment with clear aligners than those who received orthodontic treatment with traditional fixed appliances (e.g., braces) (Table 2). The number of extractions of premolars and the number of impacted teeth was also smaller in the group treated with clear aligners, but there was no significant difference between the two groups (Table 2).

Table 2. Preoperative orthodontic period, number of premolar extraction teeth, and number of impacted teeth between clear aligner group and traditional fixed appliance (e.g., bracket) group.

\begin{tabular}{|c|c|c|c|c|c|}
\hline & \multicolumn{2}{|c|}{ Clear Aligners $(n=5)$} & \multicolumn{2}{|c|}{ Braces $(n=10)$} & \multirow{2}{*}{$p$-Value * } \\
\hline & Mean & SD & Mean & SD & \\
\hline $\begin{array}{l}\text { Preoperative orthodontic } \\
\text { treatment period (month) }\end{array}$ & 11.6 & 3.01 & 15.4 & 7.86 & 0.206 \\
\hline Extracted premolar teeth (ea.) & 0.4 & 0.8 & 1.6 & 1.50 & 0.206 \\
\hline Impacted teeth (ea.) & 0 & 0 & 0.8 & 1.32 & 0.371 \\
\hline
\end{tabular}

${ }^{*}$ Fisher's exact test. If $p$-value $<0.05$, there is a significant difference between the two groups.

To evaluate postoperative stability, six measurement variables were compared between the two groups, but no significant difference was found (Table 3).

Table 3. Postoperative stability measurement between the clear aligner group and the traditional fixed appliance (e.g., bracket) group on the 2nd day and 6th month postoperatively.

\begin{tabular}{cccccc}
\hline \multirow{2}{*}{$\Delta$ T2-T1 } & \multicolumn{2}{c}{ Clear Aligners $(\boldsymbol{n}=\mathbf{5})$} & \multicolumn{2}{c}{ Braces $(\boldsymbol{n}=\mathbf{1 0})$} & \multicolumn{2}{c}{$\boldsymbol{p}$-Value } \\
\cline { 2 - 5 } & Mean & SD & Mean & 1.46 & 0.690 \\
\hline L1 to NB & 0.29 & 0.75 & 0.07 & 0.77 & 0.440 \\
\hline Pog. to NB & -0.26 & 1.03 & -0.12 & 1.74 & 0.310 \\
\hline A-Pog. & -0.52 & 1.51 & -0.92 & 1.50 & 0.440 \\
\hline AFC & -2.18 & 1.70 & 2.00 & 4.90 & 1 \\
\hline SN Vr. & 2.68 & 1.31 & 3.74 & 7.98 & 0.768 \\
\hline 0-Med. & 2.21 & 2.47 &
\end{tabular}

T1: 2nd day after surgery, T2: 6th month after surgery. ${ }^{*}$ Mann-Whitney test. $p$-value $<0.05$ indicates a significant difference between the two groups.

\section{Discussion}

There have been many studies on stability after orthognathic surgery $[7,8]$. However, there are no studies to identify the characteristics of patients who underwent orthognathic surgery after preoperative orthodontic surgery with clear aligners. There have been few reports of patients who underwent orthognathic surgery with preoperative correction with clear aligners. For example, when searching for "“clear aligner\$" and "orthognathic $\$$ "” in PubMed, there were only five papers. Among these studies, two $[9,10]$ were on the use of a stent as a clear aligner during surgery, and one [11] was on the surgery-first technique. A similar search on PubMed for "“invisalign\$" and "orthognathic\$" " only yielded four papers. 
According to the results of the study, the number of extracted premolars was fewer in the group that received preoperative orthodontic treatment with clear aligners compared to the group that had been corrected preoperatively with conventional orthodontic devices. There was no significant difference in the period of orthodontic treatment. Referring to Table 4, the average mandibular setback amount of the patients in the clear aligners group was $7.9 \mathrm{~mm}$, which showed that preoperative orthodontic treatment with clear aligners was possible even during orthognathic surgery with a relatively large setback.

Table 4. Information on five patients with clear aligners.

\begin{tabular}{|c|c|c|c|c|c|c|c|}
\hline & Sex & Age & $\begin{array}{c}\text { Preoperative } \\
\text { Orthodontic } \\
\text { Treatment Period } \\
\text { (Months) }\end{array}$ & $\begin{array}{c}\text { Extracted } \\
\text { Premolar } \\
\text { Teeth (ea.) }\end{array}$ & $\begin{array}{l}\text { Impacted } \\
\text { Teeth (ea.) }\end{array}$ & $\begin{array}{c}\text { Amount of Surgical } \\
\text { Movement }\end{array}$ & Plate Removal \\
\hline 1 & Male & 24 & 8 & 0 & 0 & $\begin{array}{c}\text { Vertical reduction } \\
(3.5 \mathrm{~mm}) \\
\text { Le Fort I osteotomy, } \\
\text { Mn. setback BSSO } \\
\text { (Rt.: } 10 \mathrm{~mm}, \text { Lt.: } 9 \mathrm{~mm})\end{array}$ & $\begin{array}{l}\text { Was performed } \\
23 \text { months } \\
\text { after surgery. }\end{array}$ \\
\hline 2 & Male & 20 & 11 & 0 & 0 & $\begin{array}{c}\text { Mn. setback BSSO } \\
\text { (Rt.: } 12 \mathrm{~mm} \text {, Lt.: } 5 \mathrm{~mm} \text { ) }\end{array}$ & $\begin{array}{l}\text { Was performed } \\
5 \text { months } \\
\text { after surgery. }\end{array}$ \\
\hline 3 & Male & 23 & 12 & 2 & 0 & $\begin{array}{c}\text { Mn. setback BSSO } \\
\text { (Rt.: } 6 \mathrm{~mm} \text {, Lt.: } 1 \mathrm{~mm} \text { ) }\end{array}$ & $\begin{array}{l}\text { Was performed } \\
11 \text { months } \\
\text { after surgery. }\end{array}$ \\
\hline 4 & Male & 22 & 10 & 0 & 0 & $\begin{array}{c}\text { Mn. setback BSSO } \\
\text { (Rt.: } 9 \mathrm{~mm}, \text { Lt.: } 11 \mathrm{~mm} \text { ) }\end{array}$ & $\begin{array}{l}\text { Was performed } \\
4 \text { months } \\
\text { after surgery. }\end{array}$ \\
\hline 5 & Male & 22 & 17 & 0 & 0 & $\begin{array}{l}\text { Mn. setback BSSO } \\
\text { (Rt.: } 9 \text { mm, Lt.: } 7 \text { mm) }\end{array}$ & $\begin{array}{l}\text { Was performed } \\
8 \text { months } \\
\text { after surgery. }\end{array}$ \\
\hline
\end{tabular}

(Mn.: mandible, BSSO: bilateral sagittal split osteotomy, Rt.: right, Lt.: left).

For maxillomandibular fixation using elastics after orthognathic surgery in our hospital, from immediately after surgery to one week after surgery, it is not possible to open the mouth at all while occluded with $4.6 \mathrm{~mm}(170.1 \mathrm{~g})$ elastic. Up to one month, it is possible to open the mouth with a $9.5 \mathrm{~mm}(170.1 \mathrm{~g})$ elastic. This protocol was equally applied to all patients participating in this study, and there were no differences between the clear aligner group and the traditional orthodontic device group in elastic guide postoperatively.

Even though the clear aligner was developed in 1999, it has evolved considerably, and many patients are receiving orthodontic treatment using the clear aligner approach $[3,12]$. A systematic review on orthodontic treatment using clear aligners found no significant difference in stability and occlusal characteristics compared to conventional orthodontic appliances [13]. Orthodontic treatment with clear aligners has the following advantages, including aesthetics, patient convenience, reduction in the number of visits to the dental clinic, decreased chair time, removable device, convenience in oral hygiene management, saving time required for wire replacement or bracket installation, and ease of taking impressions [2,14]. In addition, the patient is well accustomed to maintaining the device after surgery because they have experience with the device. In addition, there is no interference from braces during surgery (Figure 10), so there is no hindrance during surgery, and there is no risk of the bracket falling off during surgery. Another advantage of orthodontic treatment using clear aligners is that the brackets physically interfere with tooth brushing, making oral hygiene management difficult, but clear aligners do not. These removable clear aligners can also reduce the incidence of white spot lesions on teeth [15]. In a similar vein, according to Sfondrini et al., there was no significant difference in periodontal and 
microbiological parameters in patients undergoing correction with a clear aligner for two months compared with patients who did not receive orthodontic treatment [16]. According to Kankam et al., there was no significant difference in postoperative edema in orthodontic patients treated with clear aligners [17]. On the other hand, disadvantages include the need to install a screw to anchor the elastics during surgery [18], and the patient's cooperation during orthodontic treatment is important. According to a large cohort of clear aligner therapy patients, $45.5 \%$ of patients wore the aligner for $22 \mathrm{~h}$ or more a day, while $25.7 \%$ wore it for less than $11 \mathrm{~h}$ [19]. Since orthodontic treatment is required after surgery [20], the dentist and the patient must communicate in order to cooperate with the patient, and sometimes the doctor must induce the patient to show better compliance [21]. It must be replaced with the next device according to the requirements, and there is a risk of losing the orthodontic device. Furthermore, braces may be attached during the postoperative orthodontic period.

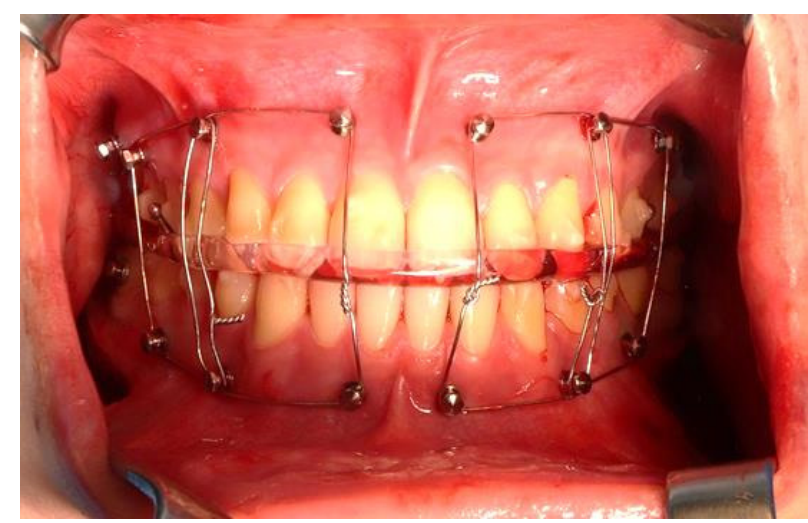

(a)

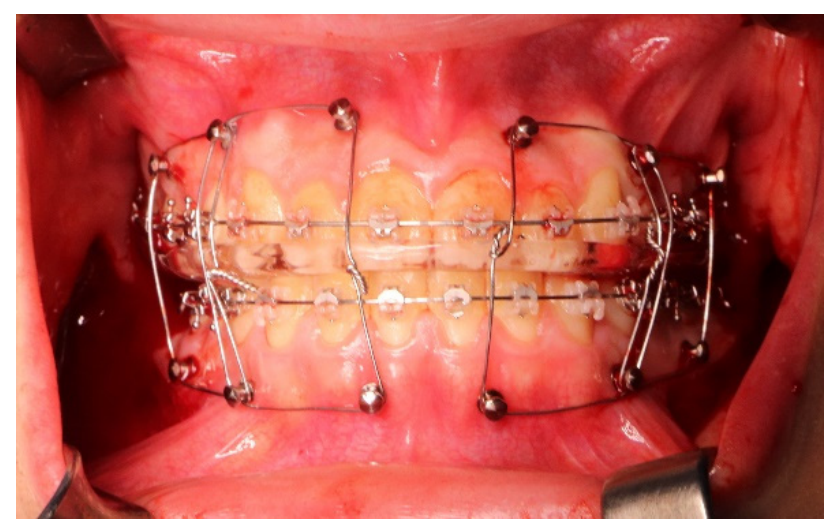

(b)

Figure 10. (a) Intraoperative clinical picture of orthognathic surgery patient with clear aligners. This photo shows the maxillomandibular fixation using wire as a final occlusion state after three mini screws were placed in each segment before fixing the miniplate to the mandible. There are no fixed braces or metal wires attached to the teeth. (b) Clinical pictures of patients who received orthodontic treatment with brackets. The photographing method is the same as in (a).

When asked about the expected disadvantages of orthognathic surgery using clear aligners, surgeons expect the preoperative period to be long, that the extraction cases will be difficult, with the possibility of using conventional fixed appliances for postoperative correction. However, in this study, there were no significant differences in the preoperative correction period in the clear aligner group. There was a case with tooth extraction and where no braces were used for postoperative correction, just clear aligners. As time goes by, orthodontists will develop orthodontic treatments using clear aligners, overcoming the limitations of orthodontics. It is thought that almost all the shortcomings can be overcome even during orthognathic surgery accompanying this clear aligner approach.

For future studies, it will be necessary to study greater numbers of patients regarding the applicability of transparent aligners to complex preoperative orthodontic treatment.

\section{Conclusions}

Even when using preoperative orthodontic treatment with clear aligners, there was no difficulty in combining clear aligners with orthognathic surgery cases. It is thought that similar results can be obtained using the combination of clear aligners with orthognathic surgery as compared with conventional surgery.

The sample size of this preliminary study was, however, limited; thus, further studies with greater numbers of cases are needed to define the role of orthognathic surgery using clear aligners. 
Author Contributions: C.M. and E.C.K. contributed to the acquisition, analysis, and interpretation of data for the work, and Y.-D.K., to the conception and design of the work, and to the analysis and interpretation. G.K.S. contributed to the interpretation of data and linguistic aspects of the manuscript. All authors have read and agreed to the published version of the manuscript.

Funding: This research received no external funding.

Institutional Review Board Statement: The study was conducted according to the guidelines of the Declaration of Helsinki, and approved by the Institutional Review Board (or Ethics Committee) of Pusan National University Dental Hospital (PNUDH-2021-0238, 17-09-21).

Informed Consent Statement: Not applicable.

Conflicts of Interest: The authors declare no conflict of interest.

\section{References}

1. Kesling, H.D. Coordinating the predetermined pattern and tooth positioner with conventional treatment. Am. J. Orthod. Oral Surg. 1946, 32, 285-293. [CrossRef]

2. Rosvall, M.D.; Fields, H.W.; Ziuchkovski, J.; Rosenstiel, S.F.; Johnston, W.M. Attractiveness, acceptability, and value of orthodontic appliances. Am. J. Orthod. Dentofacial Orthop. 2009, 135, 276 e271-212; discussion 276-277. [CrossRef] [PubMed]

3. Hennessy, J.; Al-Awadhi, E.A. Clear aligners generations and orthodontic tooth movement. J. Orthod. 2016, 43, 68-76. [CrossRef] [PubMed]

4. Kasper, F.K. 3D Printing applications in clear aligner fabrication. In Proceedings of the Forty-sixth Annual Moyers Symposium and the Forty-fourth Annual International Conference on Craniofacial Research The University of Michigan, Ann Arbor, MI, USA, 1-3 March 2019; pp. 7-21.

5. Lim, S.-H. Digital fabrication and application of clear aligner after alignment with NiTi archwires. J. Korean Dent. Assoc. 2016, 54, $551-562$.

6. Hahn, W.; Engelke, B.; Jung, K.; Dathe, H.; Fialka-Fricke, J.; Kubein-Meesenburg, D.; Sadat-Khonsari, R. Initial forces and moments delivered by removable thermoplastic appliances during rotation of an upper central incisor. Angle. Orthod. 2010, 80, 239-246. [CrossRef] [PubMed]

7. Proffit, W.R.; Turvey, T.A.; Phillips, C. Orthognathic surgery: A hierarchy of stability. Int. J. Adult. Orthodon. Orthognath. Surg. 1996, 11, 191-204. [PubMed]

8. Bailey, L.; Cevidanes, L.H.; Proffit, W.R. Stability and predictability of orthognathic surgery. Am. J. Orthod. Dentofac. Orthop. 2004, 126, 273-277. [CrossRef]

9. Border, M.; Strait, R.; Vega, L. Clear Aligner Orthognathic Splints (CAOS) and Custom Maxillary Fixation Plates for Surgery-First or Surgery-Only Cases. J. Oral Maxillofac. Surg. 2021, 79, e6-e11. [CrossRef] [PubMed]

10. Caminiti, M.; Lou, T. Clear Aligner Orthognathic Splints. J. Oral Maxillofac. Surg. 2019, 77, 1071 e1071-1071 e1078. [CrossRef] [PubMed]

11. Kook, M.S.; Kim, H.M.; Oh, H.K.; Lee, K.M. Clear Aligner Use Following Surgery-First Mandibular Prognathism Correction. J. Craniofac. Surg. 2019, 30, e544-e547. [CrossRef] [PubMed]

12. Kravitz, N.D.; Kusnoto, B.; BeGole, E.; Obrez, A.; Agran, B. How well does Invisalign work? A prospective clinical study evaluating the efficacy of tooth movement with Invisalign. Am. J. Orthod. Dentofac. Orthop. 2009, 135, 27-35. [CrossRef] [PubMed]

13. Zheng, M.; Liu, R.; Ni, Z.; Yu, Z. Efficiency, effectiveness and treatment stability of clear aligners: A systematic review and meta-analysis. Orthod. Craniofac. Res. 2017, 20, 127-133. [CrossRef]

14. Ke, Y.; Zhu, Y.; Zhu, M. A comparison of treatment effectiveness between clear aligner and fixed appliance therapies. BMC Oral Health 2019, 19, 24. [CrossRef] [PubMed]

15. Buschang, P.H.; Chastain, D.; Keylor, C.L.; Crosby, D.; Julien, K.C. Incidence of white spot lesions among patients treated with clear aligners and traditional braces. Angle. Orthod. 2019, 89, 359-364. [CrossRef] [PubMed]

16. Sfondrini, M.S.; Butera, A.; Michele, P.D.; Luccisano, C.; Ottini, B.; Sangalli, E.; Gallo, S.; Pascadopoli, M.; Gandini, P.; Scribante, A. Microbiological Changes during Orthodontic Aligner Therapy: A Prospective Clinical Trial. Appl. Sci. 2021, 11, 6758. [CrossRef]

17. Kankam, H.; Madari, S.; Sawh-Martinez, R.; Bruckman, K.C.; Steinbacher, D.M. Comparing Outcomes in Orthognathic Surgery Using Clear Aligners Versus Conventional Fixed Appliances. J. Craniofac. Surg. 2019, 30, 1488-1491. [CrossRef] [PubMed]

18. Lou, T.; Caminiti, M. Orthognathic Surgery Combined with Clear Aligner Therapy. J. Clin. Orthod. 2021, 55, 44-58. [PubMed]

19. Timm, L.H.; Farrag, G.; Baxmann, M.; Schwendicke, F. Factors Influencing Patient Compliance during Clear Aligner Therapy: A Retrospective Cohort Study. J. Clin. Med. 2021, 10, 3103. [CrossRef] [PubMed]

20. Dowling, P.A.; Espeland, L.; Krogstad, O.; Stenvik, A.; Kelly, A. Duration of orthodontic treatment involving orthognathic surgery. Int. J. Adult Orthodon. Orthognath. Surg. 1999, 14, 146-152. [PubMed]

21. Sahm, G.; Bartsch, A.; Witt, E. Reliability of patient reports on compliance. Eur. J. Orthod. 1990, 12, 438-446. [CrossRef] [PubMed] 